\title{
A survey on wireless mesh networks: architecture, specifications and challenges
}

\begin{abstract}
Wireless Mesh Network (WMN) is an emerging and practical wireless solution for metropolitan area networks to provide community broadband Internet access services by organizing and configuring nodes in the network efficiently and dynamically. In this survey, the concept of wireless mesh network has been described briefly as an emerging technology to provide Internet in large areas. Accordingly, the architecture of these networks has been explained regarding to the client meshing backbone, characteristics and advantages of wireless mesh networks have been specified, and the performance of different layers in during communication in these networks has been described. In addition, due to the performance of wireless mesh networks various issues and challenges are raised. Thus, these challenges have been specified in this paper and related research areas were proposed to solve defined challenges.
\end{abstract}

Keyword: Wireless mesh network; Mesh routers; Internet gateway; Mesh gateway 